\title{
Mechanistic insights into the synergistic activation of the RXR-PXR heterodimer by endocrine disruptor mixtures
}

\author{
V. Delfosse ${ }^{1}$, T. Huet ${ }^{1}$, D. Harrus ${ }^{1}$, M. GranelI ${ }^{1}$, M. Bourguet ${ }^{2}$, C. Gardia-Parège ${ }^{3}$, B. Chiavarina ${ }^{3}$, M. Grimaldi ${ }^{3}$, S. Le \\ Mével $^{4}$, P. Blanc ${ }^{1}$, D. Huang ${ }^{1}$, J. Gruszczyk ${ }^{1}$, B. Demeneix ${ }^{4}$, S. Cianférani ${ }^{2}$, J-B. Fini ${ }^{4}$, P. Balaguer ${ }^{3}$, W. Bourguet ${ }^{1}$ \\ ${ }^{1}$ Center for Structural Biology, Montpellier, France, ${ }^{2}$ Laboratoire de Spectrométrie de Masse Bioorganique, Strasbourg, France, \\ ${ }^{3}$ Institut de Recherche en Cancérologie, Montpellier, France, ${ }^{4}$ Muséum National d'Histoire Naturelle, Paris, France \\ william.bourguet@cbs.cnrs.fr
}

Humans are chronically exposed to mixtures of xenobiotics referred to as endocrine-disrupting chemicals (EDCs). A vast body of literature links exposure to these chemicals with increased incidences of reproductive, metabolic, or neurological disorders. Moreover, recent data demonstrate that, when used in combination, chemicals have outcomes that cannot be predicted from their individual behavior.

In its heterodimeric form with the retinoid X receptor (RXR), the pregnane $\mathrm{X}$ receptor (PXR) plays an essential role in controlling the mammalian xenobiotic response and mediates both beneficial and detrimental effects. Our previous work shed light on a mechanism by which a binary mixture of xenobiotics activates PXR in a synergistic fashion. Structural analysis revealed that mutual stabilization of the compounds within the ligand-binding pocket of PXR accounts for the enhancement of their binding affinity.

In order to identify and characterize additional active mixtures, we combined a set of cell-based, biophysical, structural, and in vivo approaches. Our study reveals features that confirm the binding promiscuity of this receptor and its ability to accommodate bipartite ligands. We reveal previously unidentified binding mechanisms involving dynamic structural transitions and covalent coupling and report four binary mixtures eliciting graded synergistic activities. Last, we demonstrate that the robust activity obtained with two synergizing PXR ligands can be enhanced further in the presence of RXR environmental ligands.

Our study [1] reveals insights as to how low-dose EDC mixtures may alter physiology and homeostasis at concentrations where individual components are considered safe. This "cocktail effect" relies on two cooperative binding processes that enhance ligand binding affinity and recruitment of transcriptional coactivators, respectively.

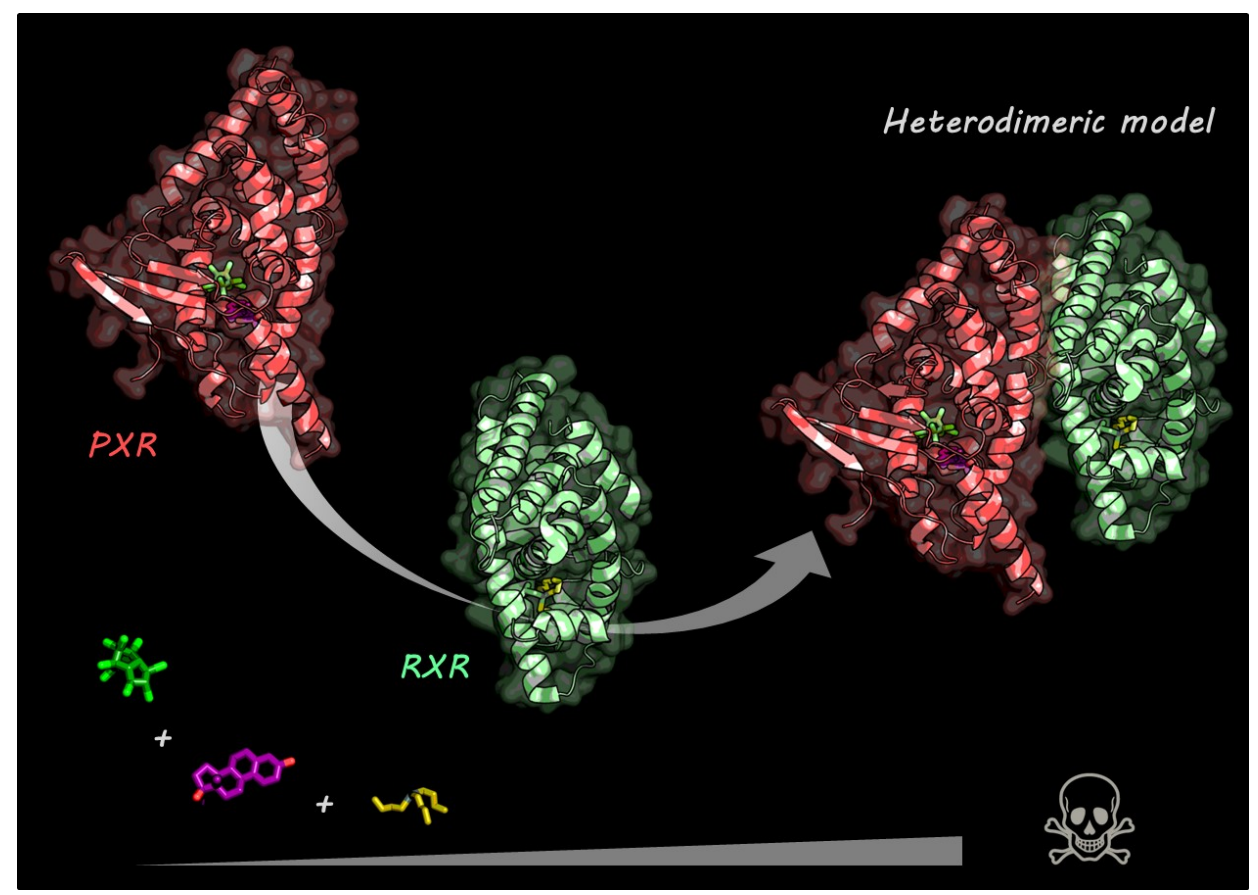

Figure 1. Synergistic activation of the RXR-PXR heterodimer by a ternary mixture of RXR (yellow) and PXR (green and violet) environmental ligands.

[1] Delfosse V, Huet T, Harrus D, Granell M, Bourguet $\mathrm{M}$, Gardia-Parège C, Chiavarina B, Grimaldi M, Le Mével S, Blanc P, Huang D, Gruszczyk J, Demeneix B, Cianférani S, Fini JB, Balaguer P \& Bourguet W. (2021). Proc Natl Acad Sci U S A. 118(1):e2020551118.

Keywords: Cocktail effect, low dose, endocrine disruptor, nuclear receptors, synergy, binding cooperativity 